\title{
Leer y escribir para no desertar en la universidad ${ }^{1}$
}

\section{Reading and writing in order not to drop out of university}

\author{
Giohanny Olave Arias ${ }^{2}$ \\ Ilene Rojas García ${ }^{3}$ \\ Mireya Cisneros Estupiñán 4
}

\section{Resumen}

Se indaga en las relaciones entre deserción universitaria y dificultades en las prácticas de lectura y escritura, de acuerdo con el panorama reciente de ambos fenómenos. Tras una revisión general de los estudios sobre deserción, donde se dimensiona su importancia para el desarrollo de la profesionalización en el país, las prácticas de comprensión y producción de textos son problematizadas en su especificidad en el ámbito de la educación superior y luego son relacionadas con el fenómeno de la deserción académica en términos causales. Finalmente, se convoca al análisis crítico de los actuales procesos de alfabetización universitaria a partir del reconocimiento de su relevancia en la misión formativa de la educación superior.

\section{Palabras clave:}

deserción universitaria, lectura y escritura en la universidad, alfabetización académica.

Abstract

This text investigates the relationships between dropping out of university and difficulties in reading and writing, in accordance with the recent overview of both phenomena. After a general review of the studies on dropping out of university, in which their importance for the continued development of Colombia's professionalization is gauged, the practices of reading comprehension and producing texts are problematized specifically in the field of higher education and are then causally related to the phenomenon of dropping out of university. Finally, the current processes of literacy at university, based on recognition of its relevance in the educational mission of higher education, are discussed.

Key words:

dropping out of university, reading and writing at university, academic literacy.

1 El artículo es derivado de la investigación titulada "En busca de la calidad educativa a partir de los procesos de lectura y escritura", inscrito en la Universidad Tecnológica de Pereira, con la dirección de Mireya Cisneros Estupiñán y con la participación de los jóvenes investigadores Giohanny Olave e llene Rojas, en la línea Lenguaje y Educación del grupo Estudios del Lenguaje y la Educación (Colciencias, A).

2 Universidad de Buenos Aires, Buenos Aires, Argentina. Correo electrónico: olavearias@gmail.com

3 Corporación Universitaria Rafael Núñez, Cartagena, Colombia. Correo electrónico: zellene7@gmail.com

4 Universidad Tecnológica de Pereira, Pereira, Colombia. Correo electrónico: mireyac@utp.edu.co 
Uno de los factores que incide de manera directa en la deserción y la mortalidad académica universitaria es el bajo nivel de comprensión y producción de textos. Tanto el abandono parcial o total de una carrera universitaria, como el desempeño insuficiente en las distintas asignaturas, se encuentran mediados por la lectura y la escritura, específicamente de textos académicos, ya que, las prácticas recurrentes en el quehacer universitario, como elaborar informes, responder exámenes, construir ensayos, preparar lecturas para la clase, y un sinnúmero de actividades más están atravesadas por la lectura y la escritura. Si bien carecemos de investigaciones centradas en la incidencia directa de estas habilidades sobre el rendimiento, deserción y mortalidad académica, las experiencias investigativas laterales permiten confirmar lo que parece ser incuestionable: buenos lectores y productores de textos obtienen mejores resultados académicos y desertan menos. Pero, ¿bajo qué especificidades se puede plantear esta relación?

\section{La deserción como problemática}

Actualmente se dispone de una multiplicidad de enfoques o modelos de análisis del fenómeno de deserción parcial y total en la teoría, que convergen en la necesidad de involucrar factores tanto de orden interno como externo al estudiante, y cuya diferencia radica en las combinaciones y énfasis de las dimensiones posibles: psicológicos, económicos, sociológicos, organizacionales e interaccionales entre estudiante-institución; el análisis de cómo estas dimensiones se combinan para proponer modelos teóricos ha sido preocupación de múltiples investigadores, destacándose Himmel (2002) y Donoso y Schiefelbein (2007). Las aproximaciones contemporáneas adquieren parámetros integradores y holísticos pues reconocen en la deserción un fenómeno complejo de múltiples causas, de impacto trascendente y de carácter mutable. Para Tinto (1989):

La cuestión de definir la deserción se desarrolla dentro del problema de la elección, es decir, en el de la identificación entre las numerosas formas de abandono que merecen una intervención. Todas las formas de abandono pueden ser rotuladas como deserción, pero no son igualmente merecedoras de acciones institucionales.

Carvajal, Trejos y Gómez (2010, p. 37), con base en las indagaciones realizadas desde la Gerencia Estratégica para la Disminución de la Deserción, de la Universidad Tecnológica de Pereira, definen el fenómeno de manera general como el proceso de abandono, voluntario o forzoso, de la carrera en la que se matricula un estudiante, por la influencia de circunstancias internas o externas a él. Así mismo, esquematizan los actores, las causas, las consecuencias, las poblaciones y las estrategias de trabajo como red extendida (Figura 1).

El esquema presenta los elementos relacionados directa e indirectamente con el fenómeno de la deserción en las universidades y, a la vez, constituye un mapa de trabajo con respecto a las acciones que deben emprenderse para intervenir el fenómeno como problema. Frente a la perceptible complejidad, una de las tareas emprendidas de manera común en universidades nacionales y extranjeras ha sido la medición estadística del fenómeno a través de estudios diacrónicos y comparativos, con predominio de análisis cuantitativos, y el seguimiento de su evolución al reconocerlo como fenómeno mutante. A nivel latinoamericano, la deserción oscila entre el $82 \%$ y el $39 \%$ por cohorte, con un promedio de $52 \%$ según cifras que pueden ser representativas de los últimos años (Figura 2).

$\mathrm{Al}$ contrastarlo con las estadísticas europeas, pese a que la Organización de Cooperación Económica y el Desarrollo (OECD) se muestra preocupada por el fenómeno en aumento al señalar que en Estados Unidos llega al 35\% en los college y en España a más del 20\% (Logros.edu, 2009), la situación de Latinoamérica sigue siendo más crítica. Al finalizar el decenio anterior, Colombia se mantuvo cercana al promedio latinoamericano con una tasa del $46,4 \%$, aunque "las cifras son inciertas, los estudios están diseminados por instituciones, no hay consolidados confiables y las políticas nacionales no son claras" (El Tiempo, 2010); cercano a este porcentaje, la Universidad de la Sabana estableció en un estudio reciente un promedio general en el país del $48 \%$, y 


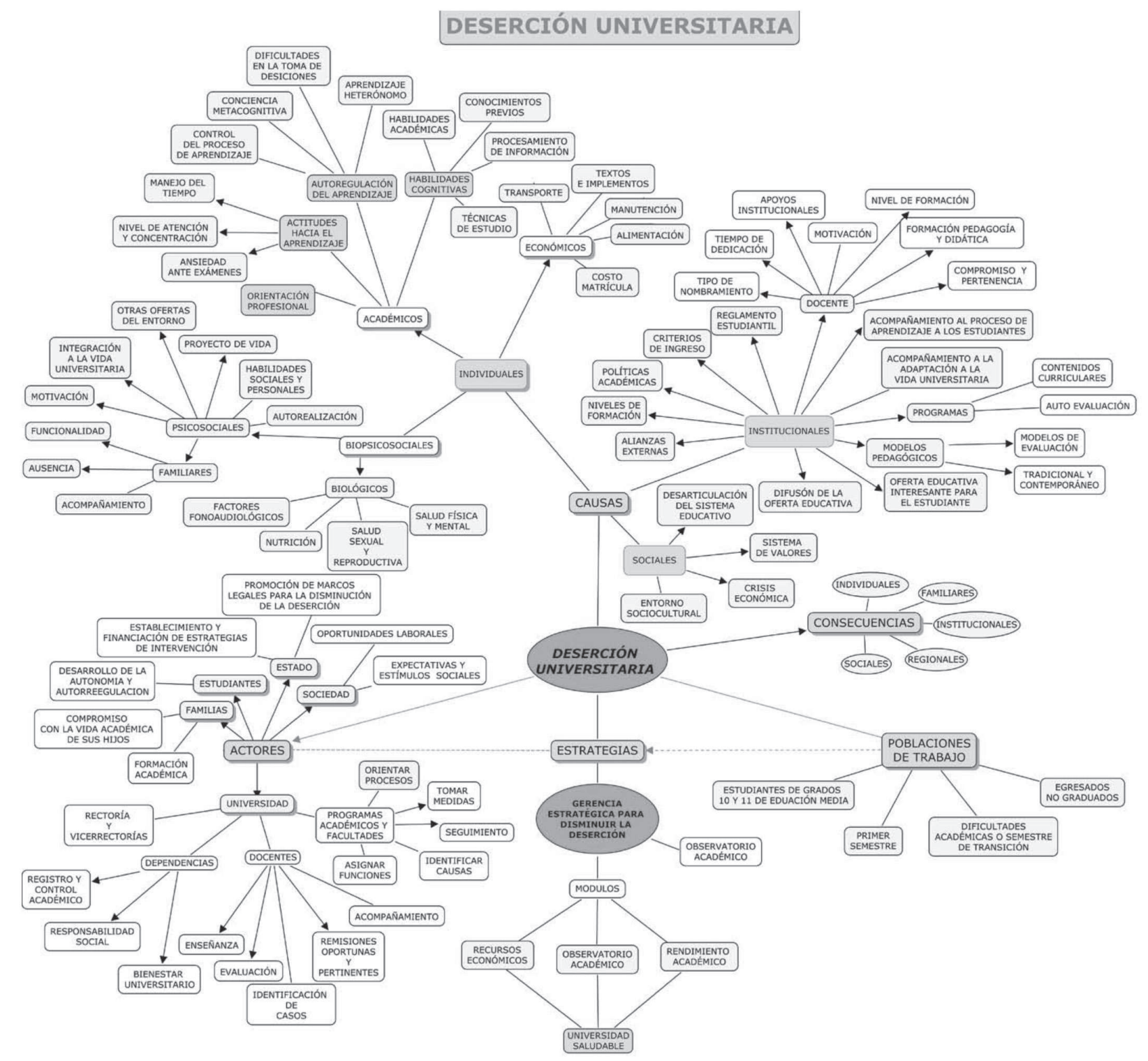

Figura 1. Deserción universitaria Tomada de Carvajal, Trejos y Gómez, 2010 (p. 37).

la Universidad de los Andes (Centro de Estudios Económicos, CEDE) del 48,2\% (Revista Dinero, 2009). El Ministerio de Educación, en asocio con esta última universidad (Sistema de Prevención y Análisis de la Deserción en las Instituciones de Educación Superior, SPADIES) ha señalado que uno de cada dos estudiantes que empiezan una carrera universitaria se retira antes de terminarla; esto incide en las metas de cobertura de educación superior que el país calcula en 50\% para el 2015, y que al terminar el 2010 se ubicó en un 35,5\%, pues según el exviceministro de Educacion Superior, Gabriel Burgos Mantilla:

El sistema de educación ha entendido que la deserción en Colombia es muy grave. No sacamos nada con ampliar el ingreso a la universidad si la mitad de los muchachos se van. Hemos iniciado un proceso de lucha contra la deserción. También nos ha abierto los ojos a un problema grave y es la calidad de los colegios porque la mayor causa de deserción viene de problemas académicos de los muchachos. (Gómez, 2009) 
Universidad Pedagógica Nacional

Facultad de Humanidades

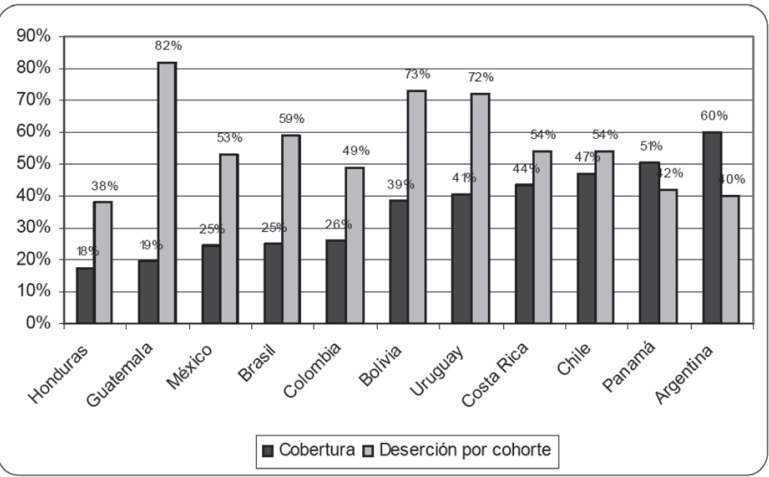

Figura 2. Deserción por cohortes en Latinoamérica Tomada de Pinto, 2007.

Al dividir en dos grandes categorías los causales de deserción, entre factores académicos y no académicos, se vislumbra el mayor peso que tiene la formación disciplinar y cognoscitiva en esta problemática. Tomando como muestra las sedes en Colombia de la Universidad Nacional para realizar la inferencia, se puede asegurar que más de la mitad de las causales de deserción universitaria responde a factores académicos. Estos datos coinciden con los de investigaciones sobre deserción a nivel institucional en la Universidad de los Andes (2002), de Antioquia (2003), La Sabana (2004), Católica (2005), Pontificia Bolivariana (2005), Los Llanos (2006), Surcolombiana (2007), de Ibagué (2008) y Tecnológica de Pereira (2010). En la Figura 3 puede observarse la proporcionalidad referida.
Isaza (2009) confirma los resultados obtenidos por la Universidad Nacional, al involucrar en el análisis porcentual el puntaje de pruebas Icfes:

Las causas académicas juegan un papel muy importante en la deserción. Así, entre los estudiantes que ingresan con mejor puntaje del Icfes, y los de menor, la diferencia en el grado de deserción alcanza el 20\%. No deja de ser preocupante que en los altos Icfes, que corresponden a un pequeño porcentaje de los bachilleres, la deserción en ellos llegue al 35\%.

A través de la actualización de los datos de CEDES y SPADIES, desde el 2004 hasta el 2009 con seguimiento a más de 2500000 estudiantes, se ha podido establecer que una mejor preparación académica en áreas fundamentales como las matemáticas y la lectura y escritura coadyuvan a que los estudiantes perseveren en las aulas; esa preparación está siendo medida por pruebas como PISA, a nivel internacional e Icfes, a nivel nacional. Según SPADIES:

Por áreas del conocimiento, la deserción es superior en las áreas como Ingeniería y Arquitectura. En contraste, el menor abandono estudiantil se registra en ciencias de la salud, debido, en gran medida, a que los estudiantes que estudian carreras, como medicina, tienen un alto examen de Estado. [...] El viceministro de Educación se

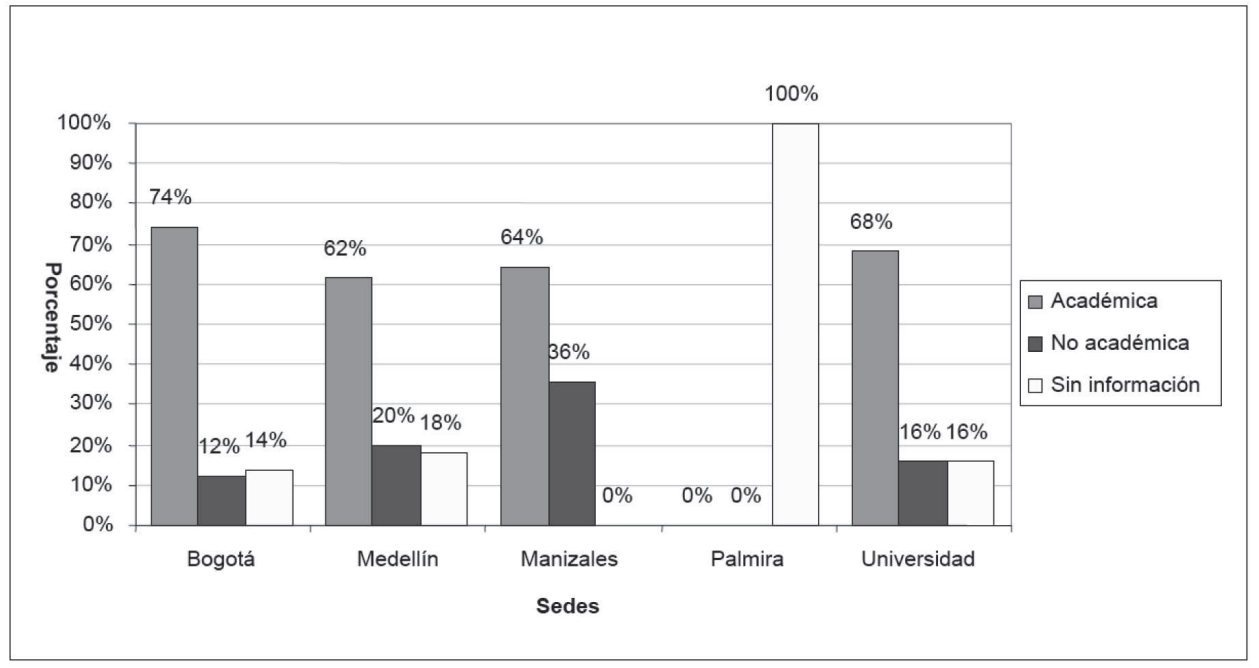

Figura 3. Deserción académica en la Universidad Nacional de Colombia Tomada de Pinto, 2007. 
mostró sorprendido por algunos de los hallazgos, como es el caso de la principal causa de deserción. Explicó que, contrario a lo que pensaba, detectar que el primer motivo de abandono estudiantil era lo académico, debido a las falencias educativas de los estudiantes en los niveles básico y secundario, específicamente en Matemáticas y Lenguaje, los ha obligado a hacer un acto de contrición. "Lo que más nos prendió las alarmas es que es un problema que viene de atrás. La mayoría de los muchachos se va, no por recursos, si no por falta de capacidad académica”, sostuvo Burgos Mantilla. (Unimedios / Universidad Nacional, 2009, p. 12)

En ese tránsito del colegio a la universidad reside un punto crítico de trabajo articulatorio en forma de preparación para los retos que se le imponen al estudiante que ingresa, en lo que Sposetti y Echavarría (2005) denominan la "dimensión educacional" propia de la universidad, que exige la construcción y deconstrucción del conocimiento. Sobre esas dificultades que devienen en mortalidad y deserción académicas, la investigadora encuentra que los alumnos "se sienten sobrepasados por la información que se les brinda en clases, y tienen problemas con la dificultad atribuida al material para estudiar, como así también a los parciales [sic]”.

\section{Deserción y prácticas textuales en la universidad}

Es clara la importancia de la lectura y la escritura en los procesos de aprendizaje y la necesidad de dominar adecuadamente ambas habilidades como herramienta de adquisición del conocimiento. Por tanto, no es posible afirmar que la tarea de enseñar a leer y a escribir es un proceso único de la escuela elemental; allí los niños adquieren el código escrito relacionándolo con un nivel de significación, pero una vez dominadas las grafías, la tarea de los agentes educativos que le siguen, incluyendo a la educación superior, se basa en desarrollar en profundidad estas habilidades.

Se trata entonces, como lo denominan Vásquez (2005) y Carlino (2005), de una alfabetización académica, un proceso educativo en el que no se enseña a los estudiantes a decodificar y dar cuenta del texto, sino a dominar estrategias de lectura avanzada que los incluyan dentro de la comunidad académica especializada de cada una de las áreas del conocimiento que les interesa.

En la educación superior, las lecturas se especializan en un área del conocimiento, lo que las hace más complejas en tanto que el estudiante no posee el dominio del código que las transversaliza. $\mathrm{Al}$ abordar estos textos, el estudiante ingresa a una comunidad de lectores que, a lo largo de la historia, ha llegado a ciertos acuerdos y ha establecido ciertos paradigmas que no requieren ser sustentados por los teóricos en cada documento que escriben, de manera que la tarea de la universidad es abrir las puertas de este grupo especializado mostrando sus paradigmas, las teorías en las que se basa la discusión y, en general, el contexto en el que se ha escrito el documento, así, el estudiante podrá comprender y aprender a partir de la lectura, reconocerá la información importante y la forma como debe abordarla e interpretarla.

Abrir el camino hacia la comprensión de textos especializados implica partir de una serie de estrategias lectoras ya adquiridas en los cursos anteriores, sin embargo, se encuentran estudiantes que ingresan e incluso permanecen en las universidades con deficiencias importantes en este campo, lo que entorpece el aprendizaje de asignaturas específicas. Ante esta situación, las universidades interrogan a la educación básica y media donde aparecen problemas similares, por lo que se cuestiona el sistema de la escuela primaria y ésta a su vez vuelve la mirada al preescolar quien registra falencias en el hogar con padres no lectores. Seguramente, para estudiantes con dificultades en lectura y escritura, el hilo conductor de una enseñanza apropiada de la lengua materna se quebró en algún punto, siendo imposible determinar cuál de todos los niveles de enseñanza es el que falló para él. La respuesta entonces parece hallarse en la necesidad de pensar el desarrollo de la comprensión lectora en todos los niveles, tanto de manera independiente como colectiva, esto porque la responsabilidad es de cada maestro, de cada escuela en particular; pero ha de ser pensada como un proceso que durará toda la vida del estudiante, 
de manera que deben tenerse en cuenta las escalas de aprendizaje para formar según las habilidades que ya adquirió y las que requiere en los años siguientes. Esto significa que la educación superior también es responsable de la formación lectora a un nivel avanzado que le permita al estudiante, no solo aprender con base en el texto escrito, sino también abrir la puerta hacia procesos de investigación y resolución de problemas.

Sin embargo, la formación en lectura y escritura en la universidad tampoco ha sido tarea fácil, al recibir estudiantes con falencias en este campo, las instituciones de educación superior tienen que hacer un doble esfuerzo: el de nivelar sus habilidades y promover aquellas que se adquieren propiamente en la universidad.

Si bien en la educación básica y media es el MEN (Ministerio de Educación Nacional) quien orienta los procesos pedagógicos al interior del aula, haciendo recomendaciones a los docentes acerca de qué y cómo enseñarlo, las universidades tienen más libertad en este sentido, según la Ley 30 de 1992, las instituciones de educación superior son autónomas para diseñar sus currículos y otros aspectos de su devenir académico, siendo vigiladas por el Icfes. Todo ello bajo un enfoque que ha de ser compartido por todas las instituciones educativas del país, en todos los niveles de educación, éste es: formar ciudadanos competitivos, reflexivos, capaces de usar el conocimiento en la resolución de problemas (Artículo 4, Ley 30 de 1992, MEN).

Bajo esta perspectiva, cada programa universitario tiene libertad para enseñar el dominio de la lengua escrita; sin embargo, en el campo de la lectura se encuentran situaciones homólogas que permiten vislumbrar un panorama acerca de lo que sucede en la universidad en este campo. Dubois afirma que "en las aulas de educación superior los profesores nos preocupamos mucho más por los textos y sus autores, cuando abordamos la lectura, que por el lector" (Dubois, 2006, p. 148). Los docentes se encuentran más atentos a que el estudiante adquiera los conceptos propios de su especialidad que a la forma como lo hacen; se piensa que la lectura es una herra- mienta para abordar el contenido de las asignaturas, un proceso de transmitir información, más que un diálogo con el texto que establezca relaciones inter y transtextuales. De manera que el docente propone documentos, los socializa en clase y luego realiza una prueba donde los estudiantes demuestren su dominio del tema, pero pocas veces se detienen a analizar la forma como abordaron el texto y su aplicabilidad en situaciones reales concretas.

El docente de cada asignatura posee el domino del paradigma conceptual que permite comprender el foco de la discusión en un texto, el interés del autor y el léxico especializado que utiliza; este dominio del conocimiento le permite guiar al estudiante a través de la lectura, aun mejor de como podría hacerlo un maestro cuyo fin único sea la enseñanza de la lengua materna. Así pues, ante la disyuntiva acerca de qué debe privilegiarse en las aulas universitarias (si una serie de conceptos o desarrollar estrategias que promuevan el pensamiento), será necesario decir que es una labor conjunta: los procesos de enseñanzaaprendizaje en todos los niveles educativos deben relacionar directamente los contenidos con su funcionalidad en procesos de análisis e investigación. De lo que se trata entonces no es de sentarse a enseñarles a leer y a escribir en un nivel más avanzado, sino de modificar las prácticas pedagógicas a través de las cuales se abordan los textos de la materia; el docente ha de ser un mediador que lleve a los alumnos a establecer relaciones entre el conocimiento que ya poseen y la información nueva entregada por el texto, a formular hipótesis y confirmarlas a través de la lectura; un docente que abra espacios para la reflexión en torno al texto y se enfoque hacia procesos metacognitivos:

Entender el proceso por el cual el estudiante arriba a una determinada interpretación y guiarlo para que reflexione sobre los motivos que lo llevaron a ella, debería ser una tarea prioritaria si lo que pretendemos es educar a nuestros alumnos en lectura. (Dubois, 2006, p. 157)

A esto se agrega la propuesta de Carlino (2005) acerca de lo que deben hacer los maestros universitarios para salvar la brecha entre la escuela y la uni- 
versidad, un salto que afecta incluso a estudiantes que dominan los procesos lectores:

Enseñar los modos específicos de nuestras disciplinas sobre cómo encarar los textos, explicitando nuestros códigos de acción cognitiva sobre la bibliografía y hacer lugar en las clases a la lectura compartida, ayudando a entender lo que los textos callan porque dan por sobreentendido [...]. Los docentes de las distintas materias no están preparados para enseñar a leer y escribir en ellos [...] pero también es cierto que los especialistas en lectura y escritura tampoco están preparados para enseñar a leer y escribir en las distintas materias, ya que desconocen los desafíos conceptuales inherentes a ellas. Es preciso desarrollar un saber colectivo. (Carlino, 2005, p. 13)

Ante esta dificultad, las universidades han diseñado cursos y programado encuentros de docentes que abren la discusión en torno a la pedagogía en el aula universitaria y la importancia de la lectura en ella. Así mismo, las universidades que ofrecen maestrías en educación abordan, en algunas o varias de sus clases, los procesos de lectura y el uso de ella como herramienta de aprendizaje y desarrollo del pensamiento. En muchos de estos cursos se brinda una orientación al maestro acerca de cómo realizar su práctica docente a nivel universitario de manera que cumpla con los parámetros de una educación superior. Entre las realizaciones del maestro en el aula de clase se sugiere, por ejemplo:

Contextualizar el texto (referencia al autor, a su obra, a la inscripción de este texto en la obra del autor), explicitar el propósito de lectura (para qué leemos este texto en esta parte del programa, qué actividad/consigna se desarrollará posteriormente -si existiese-); considerar las anticipaciones que los alumnos realizaron sobre el texto (recuperar sus hipótesis en relación al título, a otros textos del autor, entre otros); establecer vinculaciones entre los conocimientos de los alumnos y los conceptos o categorías que el texto brinda; facilitar la participación de la mayoría de los alumnos (aquello que explicitan nos dará pistas de su proceso particular de construcción del sentido). (Corrado y Eizaguirre, 2003, p. 5)
Los jóvenes que ingresan a la educación superior han de enfrentar discrepancias entre los textos que abordan en el colegio y los que les serán presentados en sus estudios universitarios. Los primeros están claramente definidos y elaborados para desarrollar un tema particular, con objetivos puntuales y estudiantes de características específicas; por su naturaleza, estas lecturas pocas veces requieren saberes previos muy especializados y, al decir de Carlino (2005, p. 39), "tratan el conocimiento como ahistórico, anónimo, único, absoluto y definitivo". Pero en la universidad el estudiante se enfrenta a amplios volúmenes de información que, si bien están delimitados por el docente en relación con la sugerencia de los materiales de lectura, estos pertenecen a un extenso corpus de conocimiento del que se debe tener cierto dominio; en ellos cobra importancia el autor y la época en la que fue escrito, además del diálogo que se establece con otros autores; son textos que no han sido escritos para el estudiante sino para la comunidad académica y que requieren habilidades de clasificación, comparación, esquematización y análisis, entre otros, para ser comprendidos, siguiendo a Carlino (2005, p. 39), "son textos que dan por sabido lo que los estudiantes no saben". Esto se relaciona a su vez con el lenguaje técnico usado por los textos abordados en la universidad, situación que se explica por su carácter científico en tanto generador de nuevos conocimientos, mientras que en la escuela, los textos están pensados en su público lector, niños y jóvenes con restricciones en el dominio de conceptos especializados.

Por su carácter eminentemente pedagógico, los libros de texto poseen lecturas cortas orientadas a ejemplificar o apoyar un concepto, se componen de microrrelatos, fragmentos, noticias o artículos de poca extensión; mientras que en la educación superior se suelen leer capítulos o libros completos que den una visión global del tema y facilite la comprensión del contexto teórico, del concepto y de la obra, en la universidad se busca la construcción de herramientas teóricas que apoyen la labor investigativa y la puesta en marcha de procesos de pensamiento encaminados a la recepción crítica del conocimiento. 
Otra de las diferencias existentes entre los textos escolares y los universitarios, es el predominio del texto narrativo en la básica y el texto expositivo-argumentativo en la superior. Es claro que los esquemas de lectura no pueden ser los mismos para ambos tipos de texto, se requieren habilidades específicas; por tanto, un estudiante con buen nivel de lectura en la escuela no necesariamente tendrá fácil acceso a los textos universitarios. Es de aclarar que en los últimos años se ha dado especial importancia a otros tipos de texto en la escuela, pero también es responsabilidad de los docentes de las otras áreas trabajar en la comprensión de lectura en un dominio específico del conocimiento.

Ambos tipos textuales (predominantemente narrativos en la escuela, expositivos y argumentativos en la universidad) están inscritos en el contexto académico en tanto que se busca la construcción y asimilación de saberes; sin embargo, el carácter de los textos escolares se relaciona con lo instruccional, se orienta específicamente a un ejercicio práctico que afianza conceptos; de otro lado, en la educación superior, los textos pasan de ser instruccionales a adquirir un carácter científico puesto que busca la inserción a una comunidad con conocimientos específicos.

A esto se agrega que es común el acceso a los textos a través de fotocopias: su uso puede dificultar el avance en niveles de comprensión puesto que son textos fragmentados, generalmente no tienen la reseña bibliográfica y menos aún los elementos paratextuales que permiten realizar inferencias enunciativas (Cisneros, Olave y Rojas, 2010, p. 49): observar la portada del libro, la solapa, el nombre del o los autores, el prólogo, el índice, la estructura de los capítulos -títulos y subtítulos-, las referencias bibliográficas, las imágenes o regresar a la lectura de un capítulo del que se hace mención posteriormente; el conocimiento de estos elementos permite al lector construir una visión global del texto, identificar la intención del autor, el público al que va dirigida la obra, de manera que el fragmento cobra sentido dentro de un contexto general.

Por último, la finalidad de lectura es distinta para cada uno de los escenarios académicos; la escuela pretende formar hábitos lectores, apoyar constructos teóricos y desarrollar la comprensión lectora; la universidad, por su parte, se orienta hacia una dimensión de análisis que requiere de una cierta experiencia de lectura, según Narvaja (2006, p. 19): "se espera que [en la universidad] un alumno lea e interprete la dimensión polémica de los discursos, establezca relaciones entre texto-autor, texto-texto y texto-conocimientos previos". Las diferencias generales entre los textos que se abordan en la escuela/ colegio y los que se leen en la universidad se condensan en la Tabla 1.

Para hacer frente al cambio en el paso hacia las prácticas textuales en la educación superior, algunas universidades cuentan con acompañamientos

Tabla 1. Diferencias entre los textos escolares y los universitarios.

\begin{tabular}{|l|l|l|}
\hline Conocimiento & General & \multicolumn{1}{|c|}{ Textos escolares } \\
\hline Enunciatario & Estudiante & Particular \\
\hline Estilo & Cotidiano & Especializado \\
\hline Extensión & Corto & Técnico \\
\hline Secuencia textual & Narrativa (predominante) & Largo \\
\hline Género discursivo & Académico-instruccional & Expositiva y Argumentativa \\
\hline Paratextos & Manual escolar & Académico-científico \\
\hline Finalidad de lectura & Formación de hábitos y & Fotocopias \\
\hline
\end{tabular}


tutoriales para los estudiantes con dificultades en el campo de la lectura y la escritura; de la misma manera, es ya una constante que varias universidades ofrezcan, en los primeros semestres, y para todos los programas académicos, cursos preparatorios donde se facilite al estudiante el dominio de los textos propios de la universidad, con resultados variables que ameritan acercamientos investigativos rigurosos, especialmente porque muchas de las dificultades detectadas en el ingreso a la universidad permanecen después de años de formación en los programas académicos (Cisneros, 2010), o en otros casos, se convierten en motivo de deserción universitaria.

A pesar de la importancia del dominio de la lengua en el contexto académico y profesional, la investigación en Colombia y en América Latina ha demostrado insistentemente que los estudiantes universitarios tienen dificultades importantes en el manejo de la lectura y la escritura, en aspectos como la definición y planeación de dinámicas enunciativas, la identificación de perspectivas e influencias teóricas en los textos, la lectura relacional entre significados, el establecimiento de jerarquías entre ideas, el uso de la organización textual como herramienta de comprensión y producción, y el hábito metacognitivo y autorregulativo al abordar tareas de lectura y escritura. Las dificultades en estas habilidades se hacen más profundas al enfrentar textos científicos especializados y otros relacionados con la vida profesional y académica.

El dominio de la lectura y de la escritura, especialmente del texto expositivo-argumentativo, constituye en elemento esencial en el desarrollo de profesionales competentes para el entorno laboral del mundo globalizado, además de implicar un factor importante en la formación de personas críticas que identifiquen problemas en su entorno y planteen soluciones.

Las serias dificultades para el cumplimiento de estos objetivos académicos, se convierten en una de las principales razones por las cuales los estudiantes no logran culminar con éxito los estudios superiores; así lo concluye la Gerencia para Disminuir la
Deserción de la Universidad Tecnológica de Pereira, en estudios realizados durante los últimos dos años, donde han determinado tres factores que influyen en la deserción universitaria: económico, vocacional y académico (fig. 4), dentro de este último se cuentan las falencias en el dominio de la lengua escrita (además del conocimiento matemático); esto ocasiona que el estudiante abandone su formación universitaria en los primeros semestres al obtener bajos promedios académicos, o bien termine sus estudios pero no alcance el título que lo acredita como profesional porque no logra consolidar el trabajo de grado. De lo anterior se puede deducir que, si bien el dominio de la lengua escrita no constituye el único factor causante de la deserción en la universidad, sí es posible afirmar que es uno de los más relevantes en cuanto permea todas las áreas del conocimiento, debe transversalizar la enseñanza de todas las ciencias y es necesario en todos los campos de la vida individual y social.

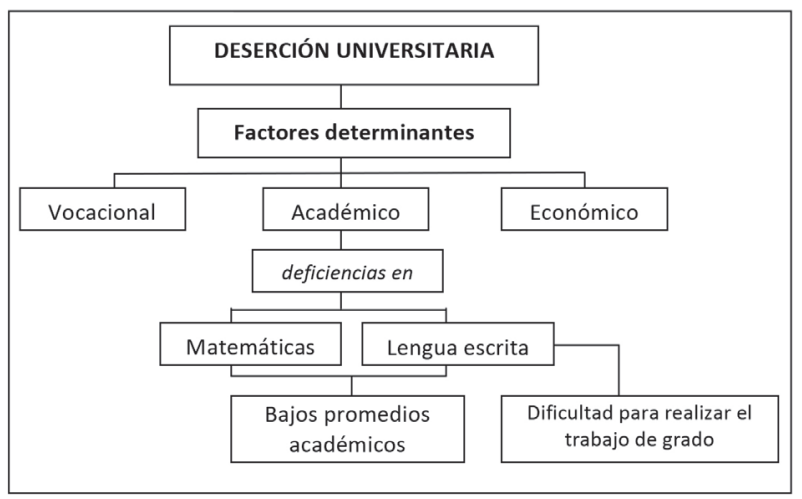

Figura 4. Factores determinantes de deserción universitaria

Basada en Carvajal, Trejos y Gómez, 2010.

El hecho de que en la universidad existan estudiantes que terminan las asignaturas pero no obtienen el título debido a las dificultades que presentan en la terminación de su proyecto de grado, demuestra que los vacíos en el desarrollo de habilidades en lectura y escritura no se quedan solo en la educación básica y media sino que continúan en la universidad; allí los estudiantes ingresan con falencias que son de difícil corrección en un nivel tan avanzado, y continúan con ellas hasta los últimos semestres de su 
Universidad Pedagógica Nacional

Facultad de Humanidades

carrera, situación que les impide avanzar en la construcción de su trabajo de grado, o bien, concluirla sin los niveles de calidad que la sociedad les demanda.

\section{Necesidad de la "alfabetización académica" en la universidad}

La función de los indicadores revisados debe ser la de alertar sobre la necesidad de alfabetizar en la universidad de manera continua, desde el primer semestre de cada programa, y apoyar estrategias para mejorar el rendimiento de los estudiantes al finalizar su etapa de educación media. Latiesa (1992, p. 50), por ejemplo, al estudiar la deserción en España, concluye que los estudiantes "no están lo suficientemente preparados para enfrentarse al mundo universitario, por deficiencias de conocimientos en ciencias básicas". Frente a la dimensión multi-causal de la deserción, las habilidades en comprensión y producción de textos se erigen como centros urgentes de dedicación académica, pues "el estudiante que posee un buen desarrollo de la aptitud verbal logra un mejor rendimiento en la Universidad" (Castañeda y Henao, 1995, p. 57), teniendo en cuenta que tal aptitud se ejerce activamente en todos los sectores disciplinares, desde las ciencias humanas hasta las ciencias exactas; así mismo, lectura y escritura en sus dimensiones comunicativa y cognoscitiva "figuran entre los motivos principales que conducen al joven universitario a desertar de sus estudios, ante la imposibilidad de procesar textos, elaborar reseñas y reportes de lectura", según investigadores de la Universidad Autónoma Metropolitana de México (Rosales, 2006).

Las tareas de lectura y escritura académica presentan sus particularidades discursivas para las cuales el estudiante novato no está ejercitado porque se trata de una inmersión a una cultura lectora y escritora universitaria que apenas inicia en su proceso de aprendizaje. Al respecto, Carlino (2005) ha insistido en la particularización de esta cultura universitaria y la necesidad de una Alfabetización académica dedicada, lo mismo que Cassany (2000, $2007,2008)$ y Parodi $(2007,2008)$ han llamado la atención sobre el papel protagónico de la compren- sión y producción de textos al interior de las profesiones, de todo lo cual se deduce la transversalidad natural de la lectura y la escritura en los programas universitarios, desde tareas comunes como las que se organizan en la Tabla 2.

En una investigación sobre las tareas de lectura y escritura que involucran los docentes universitarios en sus prácticas académicas y que causan fracaso escolar, Córdoba, Suárez y Grinsztajn (2009) encuentran que el $71 \%$ de los profesores encuestados promueve actividades en las cuales los estudiantes necesariamente deben escribir, sobre todo para la realización de informes, resúmenes y cuestionarios. El imaginario social encontrado entre la población docente habla de tres dificultades principales que afectan la consecución de esas tareas como ejercicios significativos de aprendizaje:

1. Los hábitos lectores de los estudiantes: Acostumbrados a lecturas de orden no académico, se les dificulta abordar tipologías expositivas y argumentativas propias del texto científico; además, se requiere que los estudiantes desarrollen rutinas de lectura constantes y estrategias de búsqueda y selección de datos, para que puedan abordar cantidades considerables de documentos, como es común en la academia. Aquí no se trata de entrenarse en lectura rápida sino mejor en leer estratégicamente, por ejemplo, revelando relaciones de fuerza enunciativa (Martínez, 2001, 2002, 2005, 2006) o proponiéndose objetivos críticos de lectura (Cassany, 2000, 2007,2008 ). Es importante asumir la responsabilidad docente de formar esos hábitos lectores para permitirle al estudiante disponer de distintas estrategias de comprensión textual que pueda usar de acuerdo con las necesidades específicas.

2. La divergencia de los constructos teóricos sobre lectura y escritura entre docentes y estudiantes: No se atiende con suficiente importancia al hecho de que docentes y estudiantes divergen en sus concepciones sobre la validez de los textos; de hecho, validarlos en función 
Tabla 2. Tareas de lectura y escritura académica en la universidad.

Lectura
Leer un texto para discutirlo en clase aplicando técnicas de
grupo (panel, mesa redonda, foro, etc.).

Leer un texto para ser evaluado de manera oral o escrita (quiz, controles, parciales, finales).

Realizar consultas bibliográficas en diversas fuentes y soportes (biblioteca, internet, bases de datos), de manera individual o comparativa entre perspectivas, enfoques y autores.

Leer libros o capítulos de libros como material de apoyo para asignaturas.

Leer problemas y planear respuestas a partir de planteamientos o situaciones (talleres, ejercicios extra o intraclase).

Leer enunciados interrogativos y tipos de preguntas abiertas y cerradas en situaciones de evaluación.

Leer gráficos, tablas, esquemas, mapas, infografías.

Leer cartas de solicitud, de invitación, de agradecimiento.

Leer anuncios de prensa, de revista, de volante, de afiche, de muro.

Leer tutoriales y manuales para utilizar diferentes herramientas técnicas, electrónicas o tecnológicas.

Actualizar datos y conocimientos con base en la lectura de estados del arte, antecedentes e investigaciones en curso.

\section{Escritura}

Tomar notas en clase o eventos públicos (conferencias, exposiciones, etc.).

Redactar actas y protocolos.

Redactar comentarios de texto, informes de laboratorio, informes ejecutivos, resúmenes, abstracts, palabras clave.

Redactar bibliografías comentadas, reseñas, diarios de campo, seguimientos.

Redactar reseñas reconstructivas, ensayos, artículos de opinión.

Redactar párrafos-solución a problemas retomando un planteamiento o situación.

Responder expositiva o argumentativamente en situaciones de evaluación a preguntas abiertas, o justificar la selección en preguntas cerradas.

Construir gráficos, tablas, esquemas, mapas, infografías o deducir conclusiones a partir de ellos.

Redactar cartas de solicitud, de invitación, de agradecimiento.

Redactar anuncios de prensa, de revista, de volante, de afiche, de muro.

Construir blogs y páginas web para difundir información académica.

Redactar artículos de divulgación científica o informes de investigación.

Redactar monografías de grado y tesis.

de dicotomías como "bien/mal escrito" presenta diferencias de perspectiva ante otras validaciones como "efectivo/no efectivo" o "estratégico/poco estratégico", sobre todo frente a la opinión recurrente del estudiante de que "si se entiende la idea" entonces no se necesitan otros detalles formales de estilo. Esto le dificulta al docente la tarea de sugerir correcciones, y los estudiantes pueden llegar a negarse a la reescritura por considerar que la apreciación del docente es "exagerada", "preciosista" o "perfeccionista". El origen de este tipo de resistencias se encuentra en el concepto previo que tengan docentes y estudiantes sobre la lectura y la escritura, bases empíricas y teóricas que deben ser conocidas por ambas partes antes de iniciar procesos de enseñanza-aprendizaje que involucren directamente comprensión y producción de textos.

3. La evaluación: Es frecuente que las tareas de lectura y escritura se realicen con objetivos exclusivamente evaluativos, lo cual constituye una reducción perniciosa del leer y escribir en la universidad, pues descontextualiza de la realidad estas prácticas más orientadas hacia el quehacer profesional cotidiano. Ya que en la evaluación se evidencian de manera más dramática las dificultades en lectura y escritura, es necesario pensar en la inclusión de estas habilidades como procesos, antes que como verificación de lo aprendido: "La evaluación entra en el foco de la problemática no solo por 
ser primer factor donde se evidencia la falta de alfabetización estudiantil sino que las fallas en éstas son causa de la deserción del alumnado". (Córdoba, Suárez y Grinsztajn, 2009, p. 8)

También para Rosales (2006), una causa directa de la deserción y mortalidad académica es la manera como los docentes utilizan la lectura y la escritura de manera instrumental en el proceso evaluativo, pues es costumbre que en los exámenes de conocimiento se le solicite al estudiante repetir teorías, conceptos y datos de manera memorística. Sin embargo, diagnosticar y transformar estas prácticas al interior del aula no es sencillo, por la resistencia natural de muchos docentes a evaluar sus propios métodos y, aun logrando esa apertura, por el desconocimiento sobre el cómo hacerlo. Para Romo y Fresán (2001, p. 19)

En la educación superior, el problema de la evaluación de los aprendizajes se ha discutido aún menos que en los niveles precedentes. Por lo común, se enfoca a la asignación de notas y calificaciones con criterios que tienden a simplificar la realidad evaluada y que esencialmente reflejan el conocimiento de contenidos.

Especialmente en las disciplinas desligadas de la pedagogía, lo más común es que sean los profesionales sin formación en didáctica los encargados de dictar clases, privilegiando su experiencia a nivel profesional en el ámbito empresarial o industrial, pero raras veces en el académico o el investigativo $y$, por lo tanto, desconociendo la complejidad de la evaluación y reduciéndola a la medición o utilizándola como instrumento de poder, desde imaginarios comunes como la falsa correspondencia entre la calidad del docente y la dificultad de los estudiantes para resolver sus exámenes. Esto deviene en que las prácticas de enseñanza-aprendizaje perpetúen paradigmas que intentan superar desde hace tiempo los licenciados y profesionales en pedagogía; lidiar con esta falta de formación en alfabetización académica es tarea urgente de las universidades.

González y Vela (2009) también han llamado la atención sobre los modos evaluativos en que se involucra la lectura y la escritura en la universidad; a través de la observación de estas prácticas en la Universidad Sergio Arboleda, se pueden extrapolar como lugares comunes las siguientes actividades:

- Lectura de documentos fragmentarios seleccionados por el docente, con plazos determinados para "socializar" en clase, lo que se traduce en cuestionarios orales y escritos, quiz o falsas discusiones en las cuales el docente o los estudiantes recitan el texto de nuevo.

- Escritura de trabajos finales por encargo del docente, sin proceso de elaboración y reescritura al interior de la clase, y entrega de esos trabajos sin retroalimentación adecuada, con marcas de correcciones ininteligibles o ambiguas para el estudiante.

Estas dos prácticas generales en lectura y escritura constituyen obstáculos en los procesos de enseñanza-aprendizaje significativos de cara a la formación de profesionales idóneos. Por estar arraigadas entre la comunidad académica, se precisa que ingresen a la reflexión crítica sobre su efectividad y el peso que tienen en fenómenos como la mortalidad y la deserción universitaria.

En este sentido, confirmamos que "las deficiencias vinculadas con la lectura comprensiva y la escritura, entendidas estas como competencias básicas si no son abordadas oportunamente y en forma eficaz, pueden transformarse en un factor de frustración y posterior deserción" (Córdoba, Suárez y Grinsztajn, 2009, p. 1).

De cara a esta problemática, preguntarse por las especificidades de la relación causal entre lectura/ escritura y deserción/mortalidad, así como por las responsabilidades conjuntas de estudiantes, docentes, políticas institucionales y políticas públicas (y no exclusivamente por las deficiencias del primer actor) son puntos de quiebre para la investigación actual en alfabetización académica que, según Pérez Abril (2007, p. 13), apenas empieza a acceder a la problemática "desde el tipo de demandas de lectura o escritura que hace la universidad, y desde los dispositivos didácticos y pedagógicos que configura y promueve", procesos que definen las prácticas lectoras y escritoras dentro de la cultura académica universitaria. 


\section{Referencias}

Carlino, P. (2005). Escribir, leer y aprender en la Universidad. Una introducción a la alfabetización académica. Buenos Aires: Fondo de cultura económica.

Carvajal, P., Trejos, A. y Gómez, R. (2010). En la dirección correcta, estrategias de diagnóstico y acompañamiento: una experiencia exitosa para la permanencia estudiantil. Pereira: Publiprint.

Cassany, D. (2008). Prácticas letradas contemporáneas. México: Ríos de tinta.

Cassany, D. (2007). Afilar el lapicero. Barcelona: Anagrama.

Cassany, D. (2000). Tras las líneas. Barcelona: Anagrama.

Castañeda, L. y Henao, J. (1995). La lectura en la Universidad. Medellín: Universidad de Antioquia.

Cisneros, M. (2010). La lectura y la escritura en la formación profesional: un aporte en la búsqueda de la calidad educativa (Tesis doctoral en Ciencias de la Educación). Rudecolombia, Popayan.

Cisneros, M., Olave, G. y Rojas, I. (2010). Las inferencias en la comprensión lectora. De la teoría a la práctica en la educación superior. Pereira: Universidad Tecnológica de Pereira.

Corrado, R. y Eizaguirre, M. (2003). El profesor y las prácticas de lectura en el ámbito universitario [Congreso Latinoamericano de Educación Superior en el Siglo XXI. Universidad Nacional del Centro de la Provincia de Buenos Aires]. Revista Iberoamericana de Educación. Extraído el 18 de agosto de 2012 desde: http://www.campus-oei.org/revista/edu_sup24.htm

Córdoba, M., Suárez, B. y Grinsztajn, F. (2009). Alfabetización académica en la enseñanza de Ciencias Veterinarias: la importancia de la formación docente. Extraído el 3 de junio de 2012 desde: http://inter27. unsl.edu.ar $/$ rapes $/$ ?action $=$ detalle $\&$ from $=$ todos \&id=1175

Donoso, S., y Schiefelbein, E. (2007). Análisis de los modelos explicativos de retención de estudiantes en la universidad: una visión desde la desigualdad social. Revista Estudios pedagógicos, 1(33), 7-27. Extraído el 3 de julio de 2012 desde: http://www.scielo.cl/pdf/ estped/v33n1/art01.pdf

Dubois, M. (2006) Sobre lectura, escritura... y algo más. Buenos Aires: Textos en contexto.

Fernández, G. e Izuzquiza, M. (2008). La escritura de textos en la universidad: una responsabilidad compartida. Extraído el 21 de febrero de 2012 desde: http://www.feeye.uncu.edu.ar/web/posjornadasinve/ area3/Lengua $\% 20-\% 20$ Didactica\%20de\%20la\%20 lengua $\% 20$-\%20TICs/263\%20-\%20Fernandez $\% 20$ y\%20Izuzquiza\%20-\%20UN\%20Ctro\%20Prov\%20 Bs\%20As.pdf

Gómez, A. (Diciembre, 2009). Así entra la educación superior a la década del 10. Extraído desde: http:// www.elcolombiano.com

González, B. y Vela, J. (Octubre, 2009). Prácticas de lectura y escritura en cinco asignaturas de diferentes programas de la Universidad Sergio Arboleda. Trabajo presentado en el III Encuentro Nacional y II Internacional de la Cátedra Unesco para la Lectura y la Escritura en la educación superior [CD-ROM].

González, B. (2006). Experiencia de alfabetización académica en la Universidad Sergio Arboleda de Colombia. Extraído el 5 de enero de 2010 desde: http://servidor-opsu.tach.ula.ve/unesco/even_o/iv_curso_ ea2010/z_exp_alfab_c.pdf

Himmel, E. (2002). Modelos de análisis de la deserción estudiantil en la Educación Superior. Extraído el 16 de julio de 2012 desde: http://www.universidadtecnologica.net/tportal/portales/tp4964b0e1bk102/uploa$\mathrm{dImg} /$ File/EducacionSuperior/desercion/1_\%20 DesercionE_Himmel.pdf

Isaza, J. (Marzo, 2009). Deserción en la educación superior. Extraídoel3 dejulio de 2012 desde: http://www.elespectador.com/articulo143915-desercion-universitaria

Latiesa, M. (1992). La deserción universitaria. Madrid: Siglo XXI.

Logros.edu (2009). Deserción universitaria preocupa al mundo. Extraído el 28 de junio de 2012, desde: http://www.logros.edu.pe/noticias/actualidad/810desercion-universitaria-preocupa-al-mundo.html

Malagón, L., Calderón, C. y Soto, E. (2006). Estudio de la deserción estudiantil de los programas de pregrado de la Universidad de los Llanos (1998-2004). Extraído el 27 de marzo de 2012, desde: http://web.unillanos. edu.co/centro-de-documentacion/doc_view/33estudio-de-la-desercion-estudiantil-de-los-programas-de-pregrado-de-la-universidad-de-los-llanos. html

Martínez, M. C. (2001). Análisis del discurso y práctica pedagógica. Una propuesta para leer, escribir y aprender mejor. Argentina: Homo Sapiens.

Martínez, M. C. (2002). Lectura y escritura de textos. Perspectivas teóricas y talleres. Cali: Universidad del Valle. 
Martínez, M. C. (2005). La construcción de los procesos argumentativos en el discurso. Perspectivas teóricas y trabajos prácticos. Cali: Universidad del Valle y Cátedra Unesco.

Martínez, M. C. (2006). Curso virtual sobre comprensión y composición de textos escritos. Cali: Universidad del Valle y Cátedra Unesco.

Narvaja, E. (2006). Análisis del Discurso. Modos de abordar materiales de archivo.

Buenos Aires: Santiago Arcos.

Parodi, G. (2007). El discurso especializado escrito en el ámbito universitario y profesional: constitución de un corpus de estudio. Signos, 63(40), 147-178.

Parodi, G. (Coord.) (2008). Géneros académicos y géneros profesionales: accesos discursivos para saber $y$ hacer. Valparaíso: Pontificia Universidad Católica de Valparaíso.

Pérez-Abril, M. (2007). Prácticas de lectura y escritura para el tránsito de la secundaria a la universidad: Conceptos claves y una vía de investigación. Extraído el 27 de marzo de 2012, desde: http://es.scribd.com/ doc/61800806/Practicas-de-Lectura-y-Escritura

Pinto, M. (2007). Cuestión de supervivencia. Graduación, deserción y rezago en la Universidad Nacional de Colombia. Bogotá: Universidad Nacional de Colombia.

Revista Dinero (Octubre, 2009). Se mantiene la deserción universitaria. Extraído el 27 de marzo de 1012, desde: http://www.dinero.com/actualidad/pais/articulo/ se-mantiene-desercion-universitaria/73933

Rojas, M. y González, D. (Diciembre, 2008). Deserción estudiantil en la Universidad de Ibagué, Colombia: una lectura histórica en perspectiva cuantitativa. Revista zona próxima, 9, 70-83. Extraído el 27 de marzo de 2012, desde: http://ciruelo.uninorte. edu. co/pdf/zona_proxima/9/5_Desercion\%20estudiantil.pdf

Romo, A. y Fresán, M. (2001). Los factores curriculares y académicos relacionados con el abandono y el rezago. Extraído el 3 de julio de 2012, desde: http://webcache.googleusercontent.com/search? q=cache:xGS5BxcHxIgJ:ftp://ece.buap.mx/pub/ DOCUM_EDUCATIVOS_FCE_F_PORRAS/ PORTAFOLIOS\%2520DEL\%2520TUTOR/ Lecturas/Factores\%2520acad\%25E9micos\%2 520 en $\% 2520$ abandono\%2520y\%2520rezago. $\mathrm{doc}+\& \mathrm{~cd}=1 \& \mathrm{hl}=\mathrm{es} \& \mathrm{ct}=\mathrm{clnk} \& \mathrm{gl}=\mathrm{co}$
Rosales, R. (2006). La falta de habilidades en lecto-escritura causa deserción en universidades, afirman en la UAM. Extraído el 27 de marzo de 2012, desde: http:// www.comunicacionuniversitaria.uam.mx/boletines/ anteriores06/indice/ dic5-06-1.html

Tinto, V. (Julio-septiembre, 1989). Definir la deserción: una cuestión de perspectiva. Revista Educación Superior. Revista de la Educación Superior, 18(3)(71) (18). Extraído el 3 de julio de 2012, desde: http:// www.alfaguia.org/alfaguia/files/1342823160_52.pdf

Sánchez, G., Navarro, W. y García, A. (2007). Factores de deserción estudiantil en la Universidad Surcolombiana. Sede Neiva, 2002-2005. Neiva: Universidad Surcolombiana.

Sposetti, A. y Echeverría, H. (2005). El factor educacional como causa potencial de la deserción en primer año de la universidad. Extraído el 14 de mayo de 2012, desde: http:/www.unrc.edu.ar/publicar/cde/h21.htm

Unimedios/Universidad Nacional de Colombia (Junio, 2009). Deserción universitaria. Un flagelo para la educación superior. Revista claves para el debate público, 27. Extraído el 27 de marzo de 2012, desde: http://www.agenciadenoticias.unal.edu.co/uploads/ media/Claves_Digital_No._07.pdf

Universidad Católica de Colombia (Mayo, 2005). Deserción en la Universidad Católica de Colombia. Trabajo presentado en el Encuentro Internacional sobre deserción en educación superior. Extraído el 4 de mayo de 2012, desde: http://www.mineducacion. gov.co/1621/article-85600.html

Universidad de los Andes/CEDE (2002). Deserción en las instituciones de educación superior en Colombia. Extraído el 3 de febrero de 2012, desde http://www. ascun.org.co/foro5/pleno/cedeandes.pdf

Universidad Pontificia Bolivariana (Mayo, 2005). Programa de Acompañamiento Académico, PAC. Trabajo presentado en el Encuentro Internacional sobre deserción en educación superior. Extraído el 3 de julio de 2012, desde: http://www.mineducacion. gov.co/1621/article-85600.html

Urrea, J. (28 de octubre de 2009). Deserción universitaria en el país, que llega al 50 por ciento, un fantasma en la educación superior. El Tiempo. Extraído el 3 de julio de 2012, desde: http://www.eltiempo.com/ archivo/documento/CMS-6461851

Vásquez, J., Castaño, E., Gallón, S. y Gómez, K. (2003). Determinantes de la deserción estudiantil en la Universidad de Antioquia. Extraído el 4 
de febrero de 2012, desde: http://ciencias.udea. edu.co/documentos/reacreditacion $2 /$ anexos/ Anexo\%2039.\%20Estudio\%20de\%20deserci $\%$ C3\%B3n\%20estudiantil\%20en \% 20la\%20 Universidad/Investigaci\%C3\%B3n\%20sobre\%20 deserci\%C3\%B3n.pdf

Vásquez, A. (2005). ¿Alfabetización en la universidad?. En: Colección de Cuadernillos de actualización para pensar la Enseñanza Universitaria. Universidad Nacional de Río Cuarto, 1, (1). Disponible en: http://www.unrc. edu.ar/unrc/academica/pdf/cuadernillo01.pdf

Vélez, A. y López, D. (2004). Estrategias para vencer la deserción universitaria. Informe final de la investigación sobre las causas de deserción estudiantil en la Facultad de Comunicación Social y Periodismo de la Universidad de La Sabana, Colombia. Revista Educación y Educadores, 7. Extraído el 4 de mayo de 2012, desde: http://educacionyeducadores.unisabana.edu.co/index.php/eye/article/view/555 